\title{
On the Problem of Reconstructing a Tournament from Subtournaments ${ }^{1}$
}

\author{
By \\ Frank Harary and Ed Palmer, Ann Arbor, Mich., USA \\ With 7 Figures \\ (Received November 9, 1965)
}

\section{Introduction}

No one has ever specified an elegant complete set of invariants for a graph and indeed it is evidently an extraordinarily difficult task. It has been verified that none of the usual invariants of graphs such as the number of points and lines, connectivity, chromatic number, degree sequence or group of automorphisms place much of a restriction on the graph. In fact, Izbicki [6] has shown how to construct an infinite number of regular graphs having the same group, chromatic number and degree of regularity. Since such properties as these seem to fall far short of determining a graph, it is natural to ask the question: For a given graph $G$ and a given set $S$ of subgraphs of $G$, does the set $S$ determine $G$ ? A specialization of this question is becoming quite a well known conjecture in graph theory:

Ulam's Conjecture [8]. If $G$ and $H$ are two graphs with $p$ points $v_{i}$ and $u_{i}$ respectively $(p \geqslant 3)$ such that for all $i, G-v_{i}$ is isomorphic with $H-u_{i}$, then $G$ and $H$ are themselves isomorphic.

Consider a graph $G$ with points $v_{1}, \ldots, v_{p}$. The graph $G-v_{i}$ obtained from $G$ on removing point $v_{i}$ is denoted $G_{i}$; it consists of all the points of $G$ except $v_{i}$ and all the lines of $G$ except those incident with $v_{i}$. Kelly [7] solved Ulam's problem for trees. Harary [2] reformulated it as a problem of reconstructing $G$ from its subgraphs $G_{i}$ and derived several of the invariants of $G$ from the set $G_{i}$.

${ }^{1}$ Work supported in part by the U. S. Air Force Office of Scientific Research under grant AF-AFOSR-754-65. 
The conjecture is, of course, not true for $p=2$ but Kelly has verified by exhaustion that it holds for all graphs with $p$ points, $3 \leqslant p \leqslant 6$. We have also verified the conjecture for $p=7$.

In the rest of this article we assume that $p \geqslant 3$.

We will use constructive methods to show that the conjecture holds for all tournaments which are not strong and have $p \geqslant 5$ points. We also show that the "line version" of the conjecture holds for all tournaments. The concluding section provides a method for obtaining information about a graph from its subgraphs, in particular for obtaining the score sequence of a tournament from its subtournaments. Definitions which do not appear here be found in $[1,3,4,5]$.

\section{Line Version for Tournaments}

By definition, a tournament is an oriented complete graph. The score $s_{i}$ of point $v_{i}$ is the outdegree of $v_{i}$. In Figure 1, all the tournaments

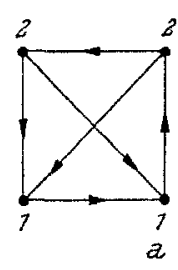

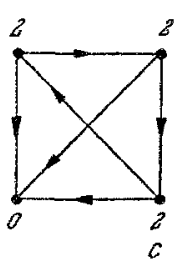

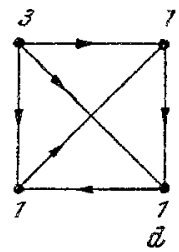

Fig. 1

with $p=4$ points are shown with the score marked at each point. It is customary to order the points $v_{i}$ so the scores satisfy $s_{1} \leqslant s_{2} \leqslant \ldots \leqslant s_{p}$.

Theorem 1. Let $T$ be a tournament with $p$ points whose lines are $x_{1}, \ldots, x_{q}$. Let $T-x_{i}$ for $i=1, \ldots, q$ be the subgraphs obtained from $T$ by deleting the line $x_{i}$. Then $T$ can be reconstructed from the $T-x_{i}$.

Proof. Clearly $T$ has a receiver if and only if some $T-x_{i}$ has a point $v$ with id $v=p-1$. If $T$ does not have a receiver, then $s_{1} \geqslant 1$ and we can choose $T-x_{i}$ with a point $u$ such that od $u=s_{1}-1$. Let $v$ be the other point of $T-x_{i}$ with total degree $p-2$. Then $T$ is obtained by adding the directed line $u v$ to $T-x_{i}$. If $T$ does have a receiver, choose $T-x_{i}$ with no points $w$ such that id $w=p-1$. 
Then $T-x_{i}$ was obtained from $T$ by deleting a line which is incident with the receiver. Let $v$ be a point of $T-x_{i}$ with id $v=p-2$ and od $v=0$. Let $u$ be the other point of $T-x_{i}$ with total degree $p-2$. Without loss of generality we can assume that $v$ was the receiver of $T$ and so $T$ is obtained by adding the directed line $u v$ to $T-x_{i}$.

\section{Point Version for Tournaments}

Again consider a tournament $T$ with points $v_{1}, \ldots, v_{p}$. Let $T_{i}=T-v_{i}$; $i=1, \ldots, p$ be the tournaments obtained by deleting $v_{i}$ from $T$. It is clear that a digraph $D$ is a tournament if and only if $D-v$ is a tournament for each point $v$ of $D$. We consider the following problem: If the collection $\left\{T_{i}\right\}$ of subtournaments is given, can $T$ be reconstructed and if so, how?

If $p=3, T$ is either a cyclic triple or a transitive triple and the collection $\left\{T_{i}\right\}$ is the same in both cases. Hence $T$ is not determined by the $T_{i}$.

If $p=4$, there are four tournaments (see Figure 1 above) and only two of them can be reconstructed. One is strong, (see Figure 1a), has score sequence $(1,1,2,2)$ and $\left\{T_{i}\right\}$ has exactly two cyclic triples. One is transitive, (Figure $1 \mathrm{~b}$ ), has score sequence $(0,1,2,3)$ and of course $\left\{T_{i}\right\}$ has no cyclic triples. The other two have score sequences $(0,2,2,2)$ and $(1,1,1,3)$ and each collection $\left\{T_{i}\right\}$ has exactly one cyclic triple. Hence in the latter two cases $T$ is not determined by the $T_{i}$.

The next theorem is not quite in the literature $[4,5]$ and will be useful in determining whether a tournament $T$ is strong, given the $T_{i}$.

Theorem 2. A tournament $T$ with at least four points is strong if and only if it has neither a transmitter nor a receiver and for some point $v, T-v$ is strong.

Proof. Suppose $T$ is strong. Clearly $T$ does not have a transmitter or a receiver. By Theorem 7 of [4], $T$ has a cycle of length $p-1$, say $Z=v_{1} v_{2} \ldots v_{p-1} v_{1}$. Therefore $T_{p}=T-v_{p}$ contains $Z$ and by Corollary 7 a of [4] it is therefore strong.

Conversely, suppose $T_{p}=T-v_{p}$ is strong. Let a complete cycle of $T_{p}$ be $Z=v_{1} v_{2} \ldots v_{p-1} v_{1}$. If $v_{p}$ is neither a transmitter nor a receiver, $v_{p}$ is adjacent to some point of $Z$ and adjacent from another. Hence by the same reasoning as in case (1) of Theorem 7 in [4], $T$ has a complete cycle and hence is strong. 
The strong tournament $T$ on three points is, of course, a cyclic triple. Each of the three subtournaments $T_{i}$ has a transmitter. That this is not the case for strong tournaments with more than three points is shown by the next theorem.

Theorem 3. If $T$ is a strong tournament with at least four points, then at most two of the subtournaments $T_{i}$ have transmitters.

Proof. Let $T$ be a strong tournament with $p \geqslant 4$ points and suppose $v_{1}$ is a transmitter in $T_{2}=T-v_{2}$. Then the score of $v_{1}$ in $T$ is at least $p-2$. Since $T$ is strong the score of $v_{1}$ is exactly $p-2$. If $T$ has two subtournaments $T_{i}$ with transmitters, there must be two points $v_{1}$ and $v_{2}$ with score $p-2$ and we can assume that $v_{2}$ is adjacent to $v_{1}$.

Since $v_{2}$ has score $p-2$, there is another point $v_{3}$ which is adjacent to $v_{2}$ and adjacent from $v_{1}$. The other $p-3$ points, $v_{i}$ with $i \geqslant 4$, are adjacent from both $v_{1}$ and $v_{2}$ (see Figure 2). Hence for $i \geqslant 4$ the score

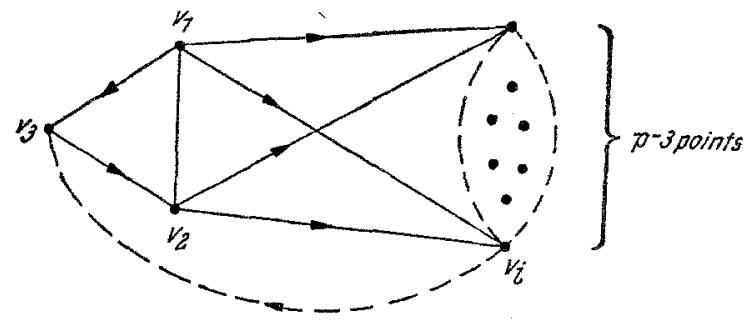

Fig. 2

of $v_{i}$ is less than $p-2$ and therefore none of these $v_{i}$ can be a transmitter in any subtournament $T-v$. Further no $T_{i}$ with $i \geqslant 4$ has a transmitter. As in Figure 2, $T_{2}$ and $T_{3}$ have transmitters $v_{1}$ and $v_{2}$ respectively. The only other possibility is that $v_{3}$ is a transmitter in $T_{1}$. But since $T$ is strong, $v_{3}$ must be adjacent from some $v_{i}$ with $i \geqslant 4$. So the score of $v_{3}$ is less than $p-2$ and $T_{1}$ does not have a transmitter.

Theorem 4. A tournament $T$ with at least five points has a transmitter if and only if at least four of the $T_{i}$ have a transmitter.

Proof. Assume that $v_{1}$ is a transmitter of $T$. Then for $i \geqslant 2, v_{1}$ is also a transmitter in $T_{i}$. Since $T$ has at least five points, there are at least four such $T_{i}$.

In the converse at least four of the $T_{i}$, say $T_{1}, T_{2}, T_{3}, T_{4}$, have a transmitter by hypothesis. Suppose $T$ does not have a transmitter. 
Let $S$ be the strong component of $T$ which is the transmitter of $T^{*}$, the condensation of $T$. Then $S$ contains at least three points. So if $v$ is any point which is not in $S$ then $T-v$ cannot have a transmitter. But then the four points $v_{1}, v_{2}, v_{3}, v_{4}$ such that $T_{i}$ has a transmitter for $i=1$ to 4 must all be in $S$. Therefore $S$ has at least four points. Further the transmitters of the $T_{i}$ are also in $S$. Thus $S-v_{i}$ has a transmitter for each $i$. Since $S$ is itself a strong tournament, this contradicts Theorem 3. Hence $T$ has a transmitter.

Corollary $4 a$. When $p \geqslant 5, T$ has a transmitter if and only if at least $p-1$ of the $T_{i}$ have a transmitter.

Note that Theorem 4 does not hold for tournaments with four points because the tournament of Figure 1d has a transmitter but only three of its subtournaments $T_{i}$ have one. Further the theorem does not hold if we only require that three of the $T_{i}$ have transmitters. If $S$, the strong component of $T$ which is the transmitter of $T^{*}$ is a cyclic triple, then three of the $T_{i}$ have transmitters but $T$ does not.

If $G$ and $H$ are two graphs with no points in common, the new graph $G+H$ is obtained by joining each point of $G$ with each point of $H$ by a line. When these additional lines are all to be directed from $G$ to $H$ we indicate this by writing $G+\rightarrow H$.
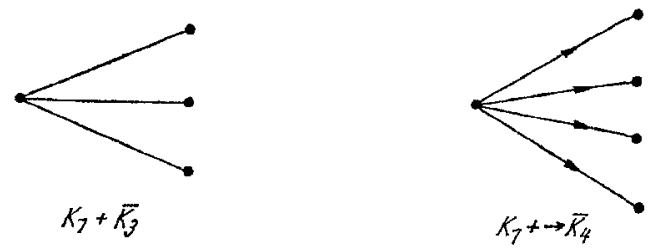

Fig. 3

Let $K_{p}$ be the complete graph on $p$ points and $\bar{K}_{p}$ its complement with $p$ points and no lines.

Here are some examples:

Theorem 5. If $T$ is a tournament with at least five points such that one of the $T_{i}$, say $T_{1}=T-v_{1}$, does not have a transmitter and at least four of the $T_{i}$ do have a transmitter, then $T=v_{1}+\rightarrow T_{1}$.

Proof. By Theorem 4, $T$ has a transmitter, say $v$. Then $T-u$ has a transmitter whenever $u \neq v$. Hence $v=v_{1}$ and $T=v_{1}+\rightarrow T_{1}$. 
Theorem 6. If $T$ is a tournament with at least five points and each $T_{i}$ has a transmitter, then $T$ can be reconstructed from the $T_{i}$.

Proof. We shall show that there is a largest integer $m$ with $2 \leqslant m \leqslant p$ such that for suitable labeling of the $T_{i}$ the following conditions hold:

(1) each $T_{i}$ has points of score $p-2, \ldots, p-m$.

(2) $T_{1}, \ldots, T_{m}$ do not have a point of score $p-(m+1)$ but $T_{m+1}, \ldots, T_{p}$ do have such a point.

(3) $T_{1}, \ldots, T_{m}$ are all isomorphic and $T=v_{1}+\rightarrow T_{1}$.

By Theorem 4, $T$ has a transmitter, say $v_{1}$. Since each $T_{i}$ has a transmitter, $T$ must bave a point, say $v_{2}$, of score $p-2$ (see Figure 4).

Now there are two possibilities:

Case 1. None of the other points $v_{i}$ with $i \geqslant 3$ has score $p-3$.

Case 2. Some point, say $v_{3}$,

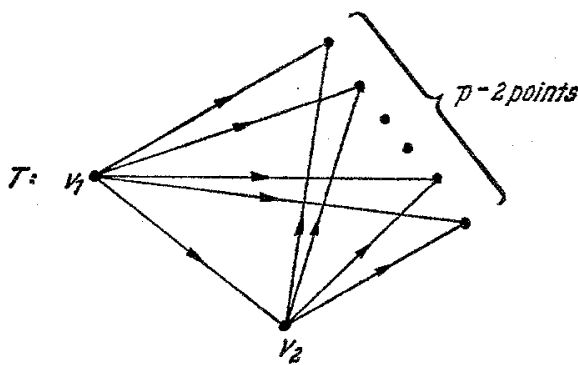

Fig, 4 has score $p-3$.

In Case $1, T_{1}$ and $T_{2}$ do not have points of score $p-3$ but for each $i \geqslant 3, T_{i}$ does (namely $v_{2}$ ). Each $T_{i}$ has a point of score $p-2$. Clearly $T_{1}$ and $T_{2}$ are isomorphic, $T=v_{1}+\rightarrow T_{1}$, and $m=2$.

In Case $2, m \geqslant 3$ and again there are two possibilities. If none of the $v_{i}$ with $i \geqslant 4$ has score $p-4$, then $T_{1}, T_{2}$ and $T_{3}$ do not have points of score $p-4$ but for $i \geqslant 4$ each $T_{i}$ does (namely $v_{3}$ ). Each $T_{i}$ has points of score $p-2$ and $p-3$. Clearly $T_{1}, T_{2}$ and $T_{3}$ are isomorphic, $T=v_{1}+\rightarrow T_{1}$ and $m=3$. Otherwise some point, say $v_{4}$, has score $p-4$ and $m \geqslant 4$. Continuing in this way we obtain (1), (2) and (3) and hence $T$ can be reconstructed. Note that if $m=p$, $T$ is the transitive tournament on $p$ points.

Every concept in directed graph theory has a "converse concept". For example the outdegree and indegree of a point are converse concepts of each other. A valuable principle mentioned in [5] is that of directional duality: For each theorem about digraphs, there is a corresponding theorem which is obtained by replacing each concept by its converse. 
Each of Theorems 3, 4, and 5 has a directional dual. The dual theorems are obtained by replacing the word transmitter by receiver.

Theorem 7. If $T$ is a tournament with at least five points and $T$ is not strong, then $T$ can be reconstructed from the $T_{i}$.

Proof. Using Theorem 4 and its dual, we can tell from the $T_{i}$ whether or not $T$ has a transmitter or a receiver. Then using Theorem 2 we can tell from the $T_{i}$ whether or not $T$ is strong. If $T$ is not strong and has a transmitter or a receiver, $T$ can be reconstructed by Theorems 5 or 6 or their directional duals.

Assume $T$ is not strong and has neither a transmitter nor a receiver. Thus $T$ must contain at least six points. Let the components of $T$ be $S_{1}, \ldots, S_{n}$ with $S_{1}$ the transmitter and $S_{2}$ the receiver in $T^{*}$. The number of points in the component $S_{i}$ is denoted by $\left|S_{i}\right|$. Since $T$ does not have a transmitter, $\left|S_{1}\right| \geqslant 3$ and since $T$ does not have a receiver, $\left|S_{2}\right| \geqslant 3$. For each $i=1, \ldots, p$ let $S_{1}^{i}, \ldots, S_{n_{i}}^{i}$ be the components of $T_{i}=T-v_{i}$ with $S_{1}^{i}$ and $S_{2}^{i}$ the transmitter and receiver respectively of $T_{i}^{*}$.

Choose the notation so that $\left|S_{1}{ }^{1}\right| \geqslant\left|S_{1}{ }^{i}\right|$ and $\left|S_{2}{ }^{2}\right| \geqslant\left|S_{2}{ }^{i}\right|$ for all $i$. Then $S_{1}$ and $S_{1}^{1}$ are isomorphic, and $S_{2}$ and $S_{2}^{2}$ are isomorphic. If $\left|S_{1}{ }^{1}\right|+\left|S_{2}{ }^{2}\right|=p$. Then $T=S_{1}{ }^{1}+\rightarrow S_{2}{ }^{2}$. Otherwise the number of components of $T$ is greater than two.

If $\left|S_{1}\right| \geqslant 4$, then by Theorem 7 of [4] there is a cycle of length $\left|S_{1}\right|-1$ in $S_{1}$. Therefore there is a point $v$ in $S_{1}$ such that $s_{1}-v$ is a strong tournament. Then we can choose $T_{3}=T-v_{3}$ with $\left|S_{1}{ }^{3}\right|=\left|S_{1}\right|-1$. Now delete all of the points of $S_{1}{ }^{3}$ from $T_{3}$ to obtain $T_{3}-S_{1}^{3}$, a subtournament

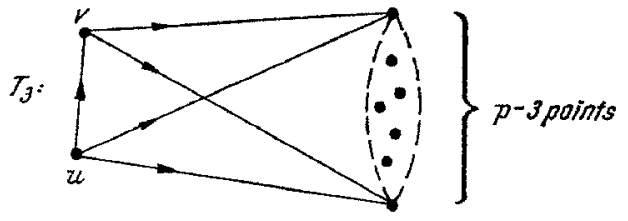

Fig. 5 of $T_{3}$. It is clear that

$$
T=S_{1}+\rightarrow\left(T_{3}-S_{1}^{3}\right) \text {. }
$$

Similarly $T$ can be reconstructed if $\left|S_{2}\right| \geqslant 4$.

$$
\text { If }\left|S_{1}\right|=\left|S_{2}\right|=3 \text {, }
$$
then both $S_{1}$ and $S_{2}$ are cyclic triples. Therefore if $v$ is a point of $S_{1}$, then $T-v$ has a transmitter. Choose $T_{3}=T-v_{3}$ so that $T_{3}$ has a transmitter. Let $u$ be the transmitter of $T_{3}$ and let $v$ be the point of $T_{3}$ which is adjacent to every point of $T_{3}$ except $u$ (see Figure 5). 
Then $T$ is obtained by adding a point $v_{3}$ to $T_{3}$ with $v_{3}$ adjacent from $v$ and $v_{3}$ adjacent to all other points of $T_{3}$ (see Figure 6).

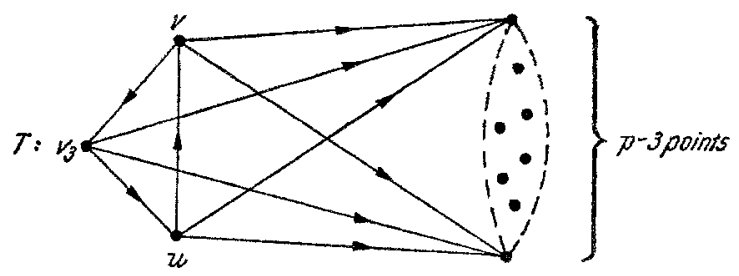

Fig. 6

\section{Remarks on Ordinary Graphs}

We now turn to ordinary graphs and prove a theorem which enables us to obtain much information about a graph $G$ from its subgraphs $G_{i}$. The following theorem is a generalization of a result which appears in $[2$, p. 48]. Note that it holds for any graph, i.e. connected or unconnected, with multiple lines or with loops, directed or undirected, etc.

Theorem 8. Let $G$ be a graph with $p>2$ points and $H$ be a connected subgraph with $n<p$ points. Let $\mu=\mu(H, G)$ be the number of subgraphs of $G$ which are isomorphic to $H$. Let $\mu_{i}=\mu\left(H, G_{i}\right)$. Then

$$
\mu=\frac{\sum_{i=1}^{p} \mu_{i}}{p-n} .
$$

Proof. Obviously $\mu_{i}=\mu-$ (the number of subgraphs of $G$ which are isomorphic to $H$ and contain $v_{i}$ ). Summing over all the $G_{i}$, we obtain

$$
\sum_{i=1}^{p} \mu_{i}=p \mu-n \mu \text {. }
$$

Corollary $8 a$. Let $T$ be a tournament with points $v_{1}, \ldots, v_{p}(p>3)$ and $T_{i}=T-v_{i}$, where the number of transitive triples in $T$ is $b=b(T)$ and the number in $T_{i}$ is $b_{i}=b\left(T_{i}\right)$. Then

$$
b=\frac{\Sigma b_{i}}{p-3} \text {. }
$$

Corollary $8 b$. Let $G$ be a connected graph with $e=e(G)$ triangles and $p>3$ points. Let each $G_{i}$ have $e_{i}=e\left(G_{i}\right)$ triangles. Then

$$
e=\frac{\sum e_{i}}{p-3}
$$


Given the collection of subgraphs $G_{i}$ the following properties of $G$ can be determined using Theorem 8 .

(1) The cycle type $\left(c_{3}, c_{4}, \ldots, c_{n}\right)$, where $c_{k}=$ the number of cycles of length $k$, provided $p>n$.

(2) The clique type $\left(f_{2}, f_{3}, \ldots, f_{n}\right)$, where $f_{k}=$ the number of complete $k$-subgraphs, provided $p>n$.

(3) The degree sequence $\left(d_{1}, d_{2}, \ldots, d_{p}\right)$, where $d_{i} \leqslant d_{i+1}$ and $d_{i}=\operatorname{deg} v_{i}$.

(4) The score sequence $\left(s_{1}, s_{2}, \ldots, s_{p}\right)$ for tournaments, where $s_{i} \leqslant s_{i+1}, s_{i}=\operatorname{od} v_{i}$ and $p-1>s_{p}$.

We outline computation of the degree sequence of a graph $G$. Let $H=K_{2}$ and let $q_{i}$ be the number of lines in $G_{i}$. Now we can apply Theorem 8 to obtain $q=\left(\Sigma q_{i}\right) /(p-2)$, the number of lines of $G$. But since $d_{i}=q-q_{i}$, for suitable relabeling we have the degree sequence $\left(d_{1}, \ldots, d_{p}\right)$ with $d_{i} \leqslant d_{i+1}$.

To compute the score sequence of a tournament, let $T$ have subtournaments $T_{i}$ as defined earlier. Theorem 4 gives a necessary and sufficient condition for $T$ to have a transmitter in terms of the $T_{i}$. Therefore when $p \geqslant 5$, we can tell from the subtournaments $T_{i}$ whether or not $T$ has a point of score $p-1$. If not, then we can choose a point $v$ in some $T_{i}$ such that the score of $v$ is greater than of equal to the score of any point of any $T_{i}$. Therefore the score of $v$ is $s_{p}$ and $s_{p}<p-1$.

Let $n_{k}$ be the number of points in $T$ of score $k$. Since the number of points in the subgraph $K_{1}+\rightarrow K_{s_{p}}$ is less than $p$, we have

$$
\begin{gathered}
n_{s_{p}}=\mu\left(K_{1}+\rightarrow \bar{K}_{s_{p}}, T\right), \\
n_{s_{p^{n-1}}}=\mu\left(K_{1}+\rightarrow \bar{K}_{s_{p}-1}, T\right)-\left(s_{p}^{s}-1\right) n_{s_{p}} .
\end{gathered}
$$

In general:

$$
n_{h}=\mu\left(K_{1}+\rightarrow \bar{K}_{k}, T\right)-\sum_{r=k+1}^{s_{p}}\left(\begin{array}{l}
7 \\
k
\end{array}\right) n_{r}
$$

In particular, when $T$ is strong, $s_{p} \leqslant p-2$ so we can always compute score sequence of $T$ from the $T_{i}$.

We conclude by observing that the score sequence and the number of cyclic triples in a strong tournament do not determine the tournament. 
In Figure 7 the two tournaments $T_{1}$ and $T_{2}$ on five points are different but each has score sequence $(1,2,2,2,3)$ and each has four cyclic triples. Clearly $T_{1}$ has a path of length two from the point of minimum score to the point of maximum score and $T_{2}$ does not.
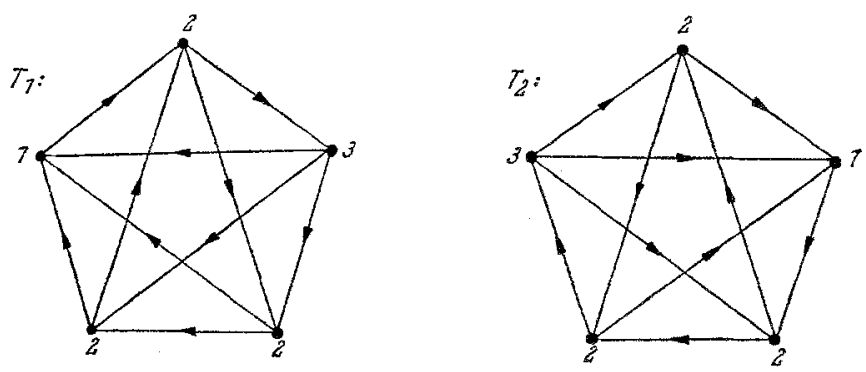

Fig. 7

It is evidently much more difficult to reconstruct the strong tournaments, but we believe that the conjecture will still be valid.

\section{References}

[1] L. Beineke and F. Harary: The maximum number of strongly connected subtournaments. Canad. Math. Bull., 8 (1965), $491-498$.

[2] F. Harary: On the reconstruction of a graph from a collection of subgraphs. A chapter in Graph Theory and its Applications, Prague, 1964, pp. $47-52$.

[3] F. Harary: The theory of tournaments: a miniature mathematical system. Colloq. Math., 15 (1966), $159-165$.

[4] $F$. Harary and $L$. Moser: The theory of round robin tournaments. Amer. Math. Monthly 73 (1966), 231-246.

[5] F. Harary, R. Norman, and D. Cartwright: Structural Models: an introduction to the theory of directed graphs. New York, 1965, esp. Chapter 11.

[6] H. Izbicki: Reguläre Graphen beliebigen Grades mit vorgegebenen Eigenschaften. Monatsh. für Math., 64 (1960), 15-21.

[7] P. J. Kelly: A congruence theorem for trees. Pacific J. Math. 7 (1957), $961-968$.

[8] S. M. Ulam: A collection of mathematical problems. New York, 1960, p. 29 .

University of Michigan. 